\title{
Preserved Covert Cognition in Noncommunicative Patients With Severe Brain Injury?
}

\author{
Caroline Schnakers, $\mathrm{PhD}^{1,2}$, Joseph T. Giacino, $\mathrm{PhD}^{3,4}$, \\ Marianne Løvstad, $\mathrm{PhD}^{5,6}$, Dina Habbal, MSc ${ }^{1,2}$, Melanie Boly, MD, PhD ${ }^{1,2}$, \\ Haibo $\mathrm{Di}, \mathrm{PhD}^{7}$, Steve Majerus, $\mathrm{PhD}^{2}$, and Steven Laureys, $\mathrm{MD}, \mathrm{PhD}^{1,2}$
}

\begin{abstract}
Background. Despite recent evidence suggesting that some severely brain-injured patients retain some capacity for topdown processing (covert cognition), the degree of sparing is unknown. Objective. Top-down attentional processing was assessed in patients in minimally conscious (MCS) and vegetative states (VS) using an active event-related potential (ERP) paradigm. Methods. A total of 26 patients were included (38 \pm 12 years old, 9 traumatic, 21 patients $>1$ year postonset): $8 \mathrm{MCS}+, 8 \mathrm{MCS}-$, and 10 VS patients. There were 14 healthy controls (30 \pm 8 years old). The ERP paradigm included (I) a passive condition and (2) an active condition, wherein the participant was instructed to voluntarily focus attention on his/her own name. In each condition, the participant's own name was presented 100 times (ie, 4 blocks of 25 stimuli). Results. In 5 MCS+ patients as well as in 3 MCS- patients and I VS patient, an enhanced P3 amplitude was observed in the active versus passive condition. Relative to controls, patients showed a response that was (I) widely distributed over frontoparietal areas and (2) not present in all blocks (3 of 4). In patients with covert cognition, the amplitude of the response was lower in frontocentral electrodes compared with controls but did not differ from that in the MCS+ group. Conclusion. The results indicate that volitional top-down attention is impaired in patients with covert cognition. Further investigation is crucially needed to better understand top-down cognitive functioning in this population because this may help refine brain-computer interface-based communication strategies.
\end{abstract}

\section{Keywords}

vegetative state, minimally conscious state, consciousness, attention, event-related potentials

\section{Introduction}

Previous neuroimaging and electrophysiological studies using active paradigms (such as mental imagery or counting) $)^{1-9}$ have reported voluntary brain activity in severely brain-injured patients who fail to respond behaviorally to command and appear to be in a vegetative state (VS; ie, eyes open with reflexive behaviors only ${ }^{10}$ or in a minimally conscious state (MCS; ie, reproducible but inconsistent volitional behaviors). ${ }^{11}$ These findings suggest the presence of covert cognition ${ }^{4,12}$ or, in other words, the presence of topdown cognitive processing in the absence of discernible motor or verbal responses. This has led to debate about the potential use of brain-computer interface (BCI) strategies to re-establish communication in this population. ${ }^{13-15}$ However, even though previous studies have shown covert cognition in some severely brain-injured patients, this does not mean that volitional top-down cognition is fully preserved. Active tasks are likely to be effortful to perform for such patients because of diminished and/or fluctuating cognitive resources.
Until now, few studies have investigated the extent to which volitional top-down cognition is functional in this type of patient. ${ }^{16}$

Different aspects of consciousness rely on distinct attentional subsystems. ${ }^{17,18}$ The tonic arousal system modulates basic wakefulness and is necessary but not sufficient for awareness. ${ }^{19}$ The phasic arousal system detects behaviorally

\footnotetext{
'University Hospital of Liège, Liège, Belgium

${ }^{2}$ University of Liège, Liège, Belgium

${ }^{3}$ Spaulding Rehabilitation Hospital and Harvard Medical School, Boston, MA, USA

${ }^{4}$ JFK Johnson Rehabilitation Institute, Edison, NJ, USA

${ }^{5}$ Sunnaas Rehabilitation Hospital, Nesoddtangen, Norway

${ }^{6}$ University of Oslo, Oslo, Norway

${ }^{7}$ Hangzhou Normal University, Hangzhou, China

Corresponding Author:

Caroline Schnakers, PhD, Coma Science Group, Cyclotron Research Centre, University of Liège and University Hospital of Liège, Sart Tilman, B30, Liège 4000, Belgium.

Email: c.schnakers@gmail.com
} 
relevant, salient, or unexpected stimuli, providing sensory awareness, and may still be active in unconscious states. ${ }^{20,21}$ Unlike the tonic and phasic arousal systems, the so-called "top-down" attentional processing is goal directed and involves volitional selection of stimuli. ${ }^{22,23}$ As an example, top-down attention can be explicitly distinguished from bottom-up attention when a person is asked to pay attention to something. Volitional top-down attention also differs from reflexive top-down attention in which attention is automatically oriented to a subset of salient stimuli (eg, cocktail party phenomenon). ${ }^{24}$ Top-down attentional control reflects a voluntary choice made to allocate resources to a specific target and represents a more demanding and sustained process than the automatic detection of salient stimuli. ${ }^{25}$ Volitional topdown attention can be investigated using event-related potentials (ERPs). In a typical oddball paradigm, the target stimulus on which the individual has to focus his or her attention is compared with the nontarget (standard) ones. The amplitude of electrophysiological response, such as the P3, is known to be modulated by attention load and is commonly higher for the target stimulus. Such a response may, therefore, be informative of the remnant attentional control in severely brain-injured patients ${ }^{26}$ and was investigated in this study.

The ability to voluntarily pay attention is essential to any cognitive activity we engage in on a daily basis. Any impairment may have a significant impact on how we deal with such activities. For severely brain-injured patients with covert cognition, such impairment could compromise the use of BCIs. A better understanding of residual cognitive functioning is hence essential to the timing and appropriate use of compensatory communication devices. In this context, we assessed and compared volitional attentional processing among patients with disorders of consciousness (DOC) using an active electrophysiological paradigm.

\section{Methods}

\section{Participants}

Inclusion and Exclusion Criteria. Patients were recruited from the University Hospital of Liege (Liège, Belgium) and the JFK Johnson Rehabilitation Institute (Edison, NJ). Medically stable VS and MCS patients (with traumatic and nontraumatic etiologies) were diagnosed using the Coma Recovery Scale-Revised (CRS-R). ${ }^{27}$ Healthy controls were matched to the patients according to handedness and age. Inclusion criteria were (1) age $>18$ years, (2) at least 28 days postinjury, (3) documented presence of auditory startle (ie, CRS-R auditory subscore $\geq 1$ ), and (4) no neuromuscular function blockers and no sedation within the prior 24 hours. Exclusion criteria were (1) documented history of prior brain injury; (2) premorbid history of hearing impairments; (3) premorbid history of developmental, psychiatric, or neurological illness resulting in documented functional disability up to the time of the injury; (4) craniectomy; (5) lobectomy; (6) open lesion of the scalp; (7) acute illness; and (8) untreated hydrocephalus.

Sample Description. In all, 30 severely brain-injured patients were recruited; 4 patients were excluded because of low quality EEG recordings (ie, ocular, muscle, and/or noise artifacts), and 26 patients ( $38 \pm 12$ years old; 18 men) were included in the ERP analyses. All patients were right handed. The etiology of brain injury was anoxic $(n=12)$, traumatic $(n=9)$, hemorrhagic stroke $(n=3)$, metabolic $(n$ $=1$ ), or encephalitis $(n=1)$. Among the patients, 19 presented a DOC for more than a year (from 1.12 to 10.4 years postinjury) and 7 for less than a year (from 34 to 363 days postinjury); 10 patients were diagnosed as being in a VS, 8 in a MCS-, and 8 in a MCS+ according to CRS-R scores (see Behavioral Assessment section below and Table 1). Three patients demonstrated either functional communication or functional object use during 1 assessment, but this performance was not observed consistently through 2 consecutive assessments and hence did not correspond to the criteria for emergence from MCS. ${ }^{11}$ In all, 15 patients were assessed at the University Hospital of Liege and 11 at the JFK Johnson Rehabilitation Institute. Using either $\chi^{2}$ tests or Mann-Whitney $U$ tests, we did not find differences between patients recruited from both centers in terms of age $(\mathrm{U}=61 ; P=.26)$, time since injury $(\mathrm{U}=71 ; P=.55)$, etiology (traumatic vs nontraumatic; $\chi^{2}=0.45 ; P=.50$ ), or diagnosis (VS vs MCS; $\chi^{2}=0.01 ; P=.90$ ). In both centers, patients were assessed by the same examiner (CS) using the same equipment. There were 14 right-handed healthy controls ( $30 \pm 8$ years old; 3 men) who also participated in the experiment. The study was approved by the ethics committee of the Faculty of Medicine of the University of Liege and by the institutional review board of the JFK Johnson Rehabilitation Institute. Written informed consent was obtained from the patients' legal surrogates.

\section{Behavioral Assessment}

The CRS-R is a sensitive and valid standardized scale that was designed to differentiate VS from MCS and that consists of 23 hierarchically arranged items that comprise 6 subscales assessing arousal, auditory, visual, motor, oromotor/verbal, and communication functions. ${ }^{27-29}$ The lowest item on each subscale represents reflexive activity, and the highest item represents cognitively mediated behaviors. The CRS-R was administered by experienced raters on the day of the EEG recording as well as several times during the week of the recording to establish the participant's diagnosis and neurobehavioral profile. Because behavioral fluctuation is common in severely brain-injured patients, ${ }^{19}$ the highest CRS-R total score obtained has been reported. 
Table I. Demographic Data for Minimally Conscious (MCS+ and MCS-) and Vegetative State (VS) Patients.

\begin{tabular}{|c|c|c|c|c|c|c|c|c|c|c|c|c|}
\hline Patient & Age (years) & Gender & Etiology & TSI & CRS-R & $\mathrm{AF}$ & VF & MF & OF & C & $\mathrm{Ar}$ & P3 \\
\hline MCS+ I & 30 & $M$ & Hemorrhagic & $9.15 y$ & 23 & 4 & 5 & 6 & 3 & 2 & 3 & I \\
\hline$M C S+2$ & 61 & $M$ & Anoxia & $0.84 \mathrm{~m}$ & 13 & 3 & 3 & 4 & 1 & 0 & 2 & I \\
\hline MCS+ 3 & 29 & $M$ & Anoxia & $1.26 y$ & 12 & 4 & I & 1 & 3 & I & 2 & 0 \\
\hline MCS +4 & 27 & $M$ & Anoxia & $4.24 y$ & 7 & 3 & 0 & 2 & 1 & 0 & I & I \\
\hline MCS+ 5 & 34 & $\mathrm{~F}$ & Hemorrhagic & $2.96 y$ & 14 & 3 & 5 & 2 & 2 & I & I & I \\
\hline MCS+ 6 & 54 & $\mathrm{~F}$ & Traumatic & $5.82 y$ & 17 & 4 & 5 & 2 & 1 & 2 & 3 & I \\
\hline MCS+ 7 & 29 & $M$ & Traumatic & $7.2 y$ & 19 & 4 & 5 & 6 & 2 & 0 & 2 & 0 \\
\hline MCS+ 8 & 47 & $M$ & Traumatic & $1.45 y$ & 14 & 3 & 5 & 2 & 1 & I & 2 & 0 \\
\hline MCS- I & 37 & $\mathrm{~F}$ & Traumatic & $10.4 y$ & 10 & I & I & 5 & 1 & 0 & 2 & 0 \\
\hline MCS- 2 & 48 & $M$ & Traumatic & $0.47 \mathrm{~m}$ & II & I & 3 & 5 & 1 & 0 & I & 0 \\
\hline MCS- 3 & 68 & $\mathrm{~F}$ & Hemorrhagic & $3.78 y$ & 7 & I & I & 3 & 1 & 0 & I & I \\
\hline MCS- 4 & 49 & $M$ & Anoxia & $1.29 y$ & 7 & 2 & 2 & 0 & 1 & 0 & 2 & 0 \\
\hline MCS- 5 & 40 & $M$ & Encephalitis & $6.52 y$ & 7 & I & 2 & 2 & I & 0 & I & 0 \\
\hline MCS- 6 & 38 & $\mathrm{~F}$ & Traumatic & $1 \mathrm{l} .7 \mathrm{~m}$ & 7 & I & 3 & I & 1 & 0 & I & I \\
\hline MCS- 7 & 39 & $M$ & Anoxia & $1.13 \mathrm{~m}$ & 9 & I & 3 & 2 & 1 & 0 & 2 & 0 \\
\hline MCS- 8 & 18 & $M$ & Anoxia & $4.06 y$ & 9 & I & 3 & 2 & 1 & I & I & I \\
\hline VS I & 28 & $\mathrm{~F}$ & Anoxia & $1.23 y$ & 4 & I & I & I & 0 & 0 & I & 0 \\
\hline VS 2 & 33 & $M$ & Anoxia & $8.29 y$ & 4 & I & 0 & 2 & 0 & 0 & I & I \\
\hline VS 3 & 40 & $M$ & Anoxia & $5.45 y$ & 7 & I & I & 2 & I & 0 & 2 & 0 \\
\hline VS 4 & 33 & $M$ & Traumatic & $2.17 \mathrm{y}$ & 5 & I & I & I & 1 & 0 & I & 0 \\
\hline VS 5 & 37 & $M$ & Anoxia & $1.13 y$ & 6 & 1 & 0 & 2 & 1 & 0 & 2 & 0 \\
\hline VS 6 & 41 & $\mathrm{~F}$ & Anoxia & 4.71 y & 4 & I & 0 & I & 1 & 0 & I & 0 \\
\hline VS 7 & 22 & $M$ & Traumatic & $6.43 \mathrm{~m}$ & 7 & I & 0 & 2 & 2 & 0 & 2 & 0 \\
\hline VS 8 & 31 & $M$ & Metabolic & $6.07 \mathrm{~m}$ & 6 & I & 0 & 2 & 1 & 0 & 2 & 0 \\
\hline VS 9 & 49 & $\mathrm{~F}$ & Anoxia & $9.37 \mathrm{~m}$ & 4 & I & 0 & I & 1 & 0 & I & 0 \\
\hline VS 10 & 31 & $M$ & Traumatic & 2.32 y & 5 & I & 0 & 2 & 1 & 0 & I & 0 \\
\hline
\end{tabular}

Abbreviations: TSI, Time Since Injury (y, years; m, months); CRS-R, Coma Recovery Scale-Revised (AF, auditory function; VF, visual function; MF, motor function; OF, oromotor function; C, communication; Ar, Arousal); P3, presence (I) or absence (0) of a higher P3 amplitude during the active versus passive condition; M/F, Male/Female; MCS, minimally conscious state; VS, vegetative state.

Based on these observations, the sample was divided into the following subgroups: $\mathrm{MCS}+$ (if any reproducible response to command was obtained; CRS-R auditory subscore $\geq 3$ ) ${ }^{30} \mathrm{MCS}-$ (if no reproducible response to command was obtained; CRS-R auditory subscore $<3$ ), ${ }^{30}$ and VS (if no localizing or voluntary response was obtained; CRS-R subscores as follows: auditory $<3$; visual $<2$; motor $<3$; oromotor $<3$; communication $<1$; arousal $<3$ ). ${ }^{10}$

\section{EEG Recordings}

EEG data were acquired at the patients' bedside. A 32-electrode skull cap (Quik-cap; http://medcat.nl) ${ }^{31}$ was connected to a portable digital EEG amplifier (NuAmps; http:// compumedicsneuroscan.com). An electro-oculogram was acquired using 2 electrodes placed diagonally above and below the right eye. A ground electrode was placed near Fz, and a nasal reference was applied. EEG data were recorded on a laptop computer. The impedances were kept below 5 $\mathrm{k} \Omega$. A sampling rate of $500 \mathrm{~Hz}$ and an analog bandpass filtering of 0.1 to $200 \mathrm{~Hz}$ were used. This procedure lasted approximately 30 minutes. Auditory stimuli were presented binaurally via earphones. EEG recordings were performed while the participants were in a wakeful state, with eyes open, in a setting with minimal ambient noise.

\section{ERP Paradigm}

We developed a single-stimulus active task designed to elicit the P3 component, which is modulated by attentional demand. ${ }^{26,32}$ In contrast to the classical oddball paradigm, the single-stimulus paradigm ${ }^{33}$ presents a target but no standard tone stimulus. Previous studies using such paradigms have shown that manipulation of the interstimulus interval, the stimulus intensity, or the instruction (press a key or count a target) produced P3 amplitudes and latencies highly similar to oddball procedures. ${ }^{33-37}$ In the current paradigm, the participant's own name was used as target stimulus because it is a subjectively meaningful stimulus that captures attention and continues to elicit a P3 even after a high number of repetitions. ${ }^{38}$ Each participant's own name was recorded with the same affectively neutral female voice and digitized for 
binaural replay during the experiment at a maximal $90-\mathrm{dB}$ sound pressure level. The paradigm consisted of the participant's own name (duration range: 500-600 ms) repeated 100 times (stimulus onset asynchrony of $2000 \mathrm{~ms}$ ) over 4 consecutive blocks (ie, 25 stimuli per block). Two conditions (each including 4 blocks) were established. The first condition was a passive one during which participants heard their own name with the following instruction: "You will hear your own name. You have nothing to do but stay awake." Thereafter, an active condition was presented, in which the same blocks were used. Participants were instructed to voluntarily focus attention on their own name with the following instruction: "This time, try to listen attentively to your own name as there might be a change in the voice pitch." There was actually no change in pitch, thus making the stimuli in the 2 conditions comparable because only the cognitive task requirements were manipulated. A break of 5 minutes was included between the passive and active conditions. The passive condition was always presented before the active one. Task instructions were repeated between each block. Brief auditory or deep-pressure stimulation was applied immediately before each block in both active and passive conditions to ensure a sufficient arousal level. The entire paradigm lasted approximately 15 minutes.

\section{ERP Analyses}

Analyses of EEG data were performed using Statistical Parametric Mapping software (SPM8; www.fil.ion.ucl. ac.uk/spm). Data were filtered between 0.1 and $20 \mathrm{~Hz}$ and epoched between $-200 \mathrm{~ms}$ prestimulus and $1300 \mathrm{~ms}$ poststimulus. A baseline correction was applied on the epoched data. Single epochs with an amplitude greater than or equal to $\pm 75 \mu \mathrm{V}$ on electro-oculogram electrodes were discarded from further analysis. By using a robust averaging (which automatically excludes artifactual time points within each trial), single-subject ERP averages were computed by condition (ie, active vs passive). A grand-averaged ERP was computed for each group (ie, control, $\mathrm{MCS}+, \mathrm{MCS}-$, and VS). Based on the grand-averaged ERPs, we determined a temporal window between 400 and 800 ms poststimulus for the P3 component. Temporal windows (poststimulus) were also defined for earlier ERP components such as the N1/P2 complex (window: 0-200 ms) and the $\mathrm{N} 2$ component (window: 200-400 ms) because these reflect basic auditory and language processing. ${ }^{26}$ Single-subject ERP averages were checked to ensure that each ERP component was included in the corresponding defined temporal window. Amplitude values (in $\mu \mathrm{V}$ ) were automatically computed at each time point of the single-subject averages in the active and passive conditions for statistical purposes.

Differences in ERP Responses Between Conditions. The amplitudes obtained within the time window of each ERP component were entered in an ANCOVA model, with group (control, MCS+, MCS-, and VS) as the between-subject factor and condition (active vs passive) as the within-subject factor. A paired $t$ test was also conducted at an individual level to detect differences of amplitude values (automatically computed on single-trial amplitudes in the 2 conditions at each time point). The proportion of responders per group was reported. SPM statistical analyses have, therefore, been done both at the group level (using ANCOVA) and at the individual level (using $t$ tests). The individual analyses have been performed to determine the group of responders on whom we would apply further analyses (see below).

Differences in ERP Responses Across Blocks. Differences of amplitude values between conditions were estimated for responders in all 4 blocks for the ERP components of interest. For each group, an ANCOVA model was applied, with condition (active vs passive) and block (1 to 4 ) as withinsubject factors and group as the between-subject factor. A paired $t$ test was also conducted for each block at an individual level (as described above).

Differences in ERP Responses Between Groups. Responders with covert cognition (CC; ie, including MCS- and VS responders) $)^{1,2,4}$ were gathered into the $\mathrm{CC}$ group to compare their ERP responses with that of responders from the MCS+ and control groups. The amplitude values obtained in responders for the active condition (within the time window defined for the ERP components of interest) were entered in an ANCOVA model, with group (ie, control, MCS+, and $\mathrm{CC})$ as the independent variable.

Responders' Demographics. A $\chi^{2}$ test was used to assess whether the proportion of responders differed according to the etiology (traumatic vs nontraumatic). Mann-Whitney $U$ tests were performed to test whether the age and the time since injury differed between responders and nonresponders. An ANCOVA model was applied, with condition (active vs passive) as the within-subject factor, to detect whether the difference of amplitude values for the ERP components of interest covaried with responders' CRS-R total scores and subscores.

We expected a modulation of the $\mathrm{P} 3$ amplitude according to condition (ie, active vs passive).$^{34}$ Patients/controls with significantly higher $\mathrm{P} 3$ amplitude values in the active vs passive condition were considered as responders. As the P3 component can typically be observed from parietal to frontal electrodes, ${ }^{26,32}$ the analyses focused on F3, Fz, F4, FC3, $\mathrm{FCz}, \mathrm{FC} 4, \mathrm{C} 3, \mathrm{Cz}, \mathrm{C} 4, \mathrm{CP} 3, \mathrm{CPz}, \mathrm{CP} 4, \mathrm{P} 3, \mathrm{Pz}$, and P4 (see supplementary material). At each electrode, $P$ values were obtained for each time point within the temporal window defined for each ERP component. Results were considered significant at $P<.05$, corrected for multiple comparisons 
using Random Field Theory (which allows one to identify locations where the amplitude values differ reliably across participants between 2 conditions at a given time point, having corrected for the multiple $t$ tests performed in space and time). The ranges of time points (or latencies) for which we obtained significant results were reported. For a comparable methodological approach (see Boly et $\mathrm{al}^{39}$ ).

\section{Results}

\section{Differences in ERP Responses Between Conditions}

The N1/P2 complex and N2 component did not differ according to the condition in any group. In contrast, significant results were obtained for the $\mathrm{P} 3$ component. The control group displayed larger $\mathrm{P} 3$ amplitudes in the active compared with the passive condition over frontocentral electrodes. This effect was more widely distributed in MCS+ patients over the parietal, central, and frontal electrodes. Enhanced P3 amplitudes were observed neither in the MCS- group nor in the VS group. At an individual level, differences were found in 11 of 14 controls (79\%); 5 of $8 \mathrm{MCS}+$ patients $(63 \%)$, but also in 3 of 8 MCS- patients (44\%); and in 1 of $10 \mathrm{VS}$ patients $(10 \%)$ for at least 1 of the electrodes included in the analyses (see Table 1 and Figure 1).

\section{Differences in ERP Responses Across Blocks}

Considering the responders, higher P3 amplitudes were obtained in the active condition in all blocks for the control group but in only 3 blocks for the MCS+ group, the MCSgroup, and for patient VS2 (see Figure 2). Taken individually, higher amplitudes were obtained for 4 blocks in all responders of the control group but also in patients MCS +1 and MCS +2 ; for 3 blocks in patients MCS $+4, \mathrm{MCS}+5$, MCS +6 and patients MCS -3 and MCS -8 ; and for 2 consecutive blocks in patient MCS- 6 .

\section{Differences in ERP Responses Between Groups}

The control group presented higher P3 amplitudes over frontocentral electrodes compared with the $\mathrm{CC}$ group and in parietal, central, and frontal electrodes compared with the MCS+ group. No significant difference was observed between the CC group and the MCS+ group (see Figure 3).

\section{Responders' Demographics}

Neither etiology $(\chi=0.93 ; P=.33)$, time since injury $(U=$ $49 ; P=.14)$, nor age $(U=74 ; P=.89)$ differed between responders and nonresponders. The difference in P3 amplitude values between conditions did not covary with responders' CRS-R total scores or CRS-R subscores.

\section{Discussion}

The main objective of this study was to explore electrophysiological indices of volitional top-down attention among patients with DOC using an active ERP paradigm. Enhanced P3 amplitudes were observed in the active versus passive condition not only in controls and in $\mathrm{MCS}+$ patients, but also in 3 of 8 MCS- patients and 1 of 11 VS patients (VS2). Because both conditions include exactly the same stimulation and only differ with regard to the instruction, a change in the ERP responses (particularly, in an ERP component known to be modulated by attention, such as the P3) ${ }^{34}$ is likely to be associated with the change of instruction and imply that the patient understood the verbal command and willfully responded to this one and, therefore, that he or she was conscious. This finding is in line with previous findings using active paradigms in patients with DOC and showing the presence of covert cognition in a minority of MCS- and VS patients. ${ }^{1-9}$ Those studies did not investigate the extent to which these occasional signs of volitional top-down cognition in responders really reflect fully functional top-down cognitive processing. Recently, Chennu et $\mathrm{al}^{16}$ investigated such processing and observed a preserved volitional topdown attention in $1 \mathrm{VS}$ patient. The number of patients with covert cognition that we had in our study allowed us to better characterize those responses to see how they differ from those of MCS+ and controls and how they fluctuate over time. Our results suggest that top-down cognitive processing in these patients is characterized by diminished and fluctuating top-down attentional control.

The controls primarily showed higher P3 amplitudes over frontocentral electrodes. This topography is usually related to one of the subcomponents of the P3, the P3a. The P3a originates from stimulus-driven attentional processing. ${ }^{26}$ Previous studies have reported a variation of the P3a amplitude according to the amount of focal attention engaged to discriminate stimuli. ${ }^{40,41}$ For MCS+ patients and patients with covert cognition (ie, MCS- and VS), higher P3 amplitude values were observed over frontal but also parietal electrodes, which may be related to the frontoparietal attention network and may indicate a need for more generalized attentional activation in severely brain-injured patients. ${ }^{42}$ The parietally distributed $\mathrm{P} 3$ has also been associated with working memory processes. ${ }^{26}$ In our paradigm, working memory may have been recruited to maintain the instruction available within each block of the active condition.

Besides the potential involvement of additional cognitive resources when performing the task, our results seem to indicate that patients' performance fluctuates. An enhanced P3 amplitude was consistently observed across the 4 blocks in controls, whereas this was observed in only ( 2 to) 3 blocks in 60\% (3 of 5) of MCS+ patients and also in all patients with covert cognition. This suggests that patients with covert cognition may have an impaired vigilance 


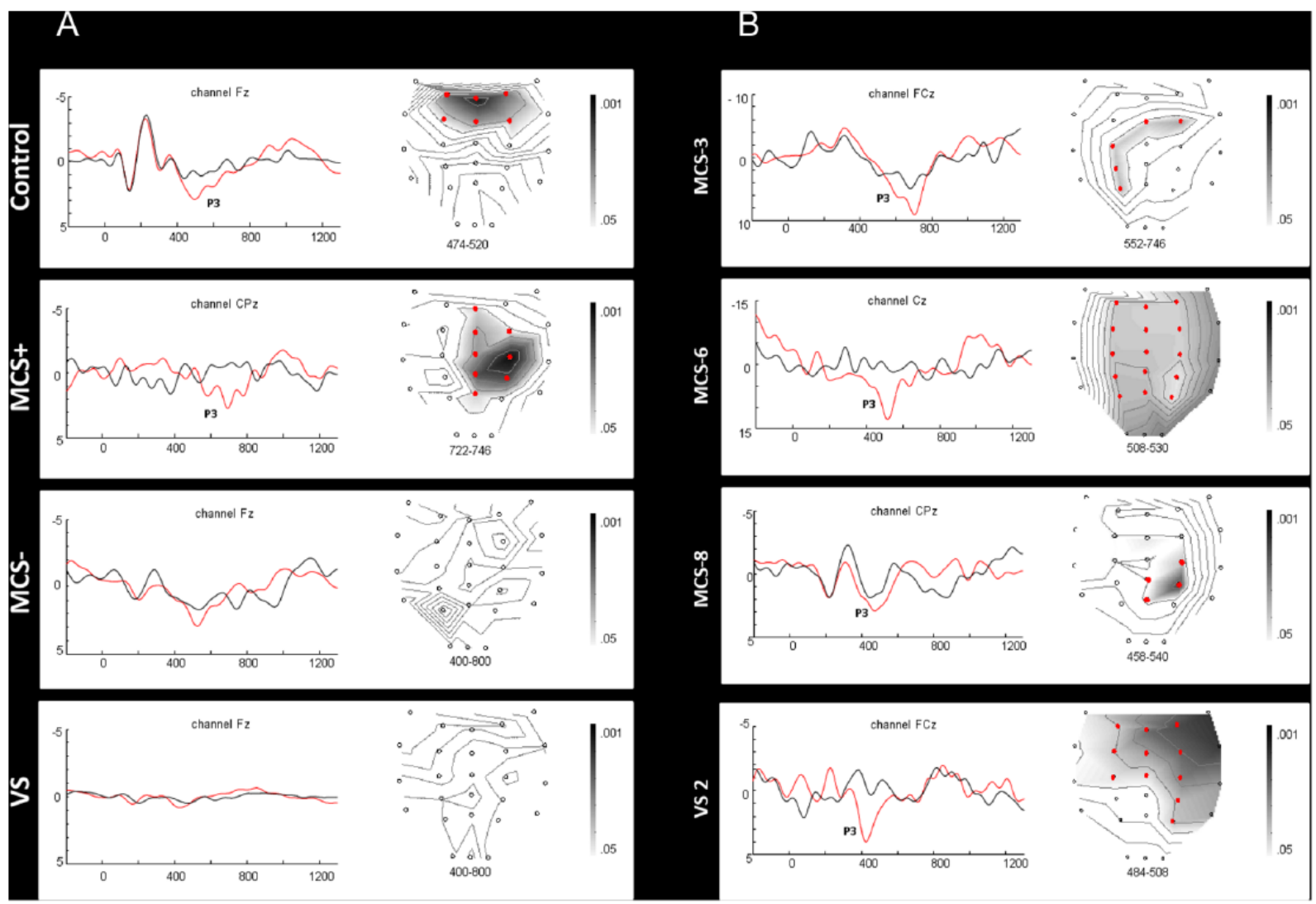

Figure I. Differences in ERP responses between conditions: panel A reports the results for each group (ie, control, MCS+, MCS-, and VS), whereas panel B reports individual results for patients with covert cognition (ie, VS2, MCS- 3, MCS- 6, and MCS- 8). The left part of each panel illustrates the averaged ERPs in the active (in red) versus passive (in black) condition ( $y$ axis, amplitude in $\mu \mathrm{V}$; $x$ axis, time in $\mathrm{ms}$ ). The right part of each panel displays statistical parametric $T$ maps for the differences of P3 amplitude between conditions ( $P$ values <.05 to .001 in gray on the map; red circles are electrodes for which a significant result was observed) within the time window of interest (in ms; mentioned under the map).

Abbreviations: ERP, event-related potential; MCS, minimally conscious state; VS, vegetative state.

similar to what is clinically observed in MCS patients. ${ }^{11}$ Patients with covert cognition also presented lower P3 amplitudes over frontocentral electrodes compared with controls, which may reflect impaired availability of attentional resources. ${ }^{26}$ In fact, P3 amplitudes did not differ between $\mathrm{MCS}+$ patients and patients with covert cognition, which may indicate similar cognitive processing.

No link was found between CRS-R total scores/subscores and P3 amplitude values. This is not surprising because we found higher amplitudes in the active condition even in MCS- and VS patients who have a low CRS-R. An absence of correlation between behavioral and electrophysiological responses during active tasks has also been observed in previous findings. ${ }^{4}$ This supports recent assumptions on the potential dissociation between behavioral expression of consciousness and consciousness per se in patients with severe acquired brain injury. ${ }^{43}$ Only a minority of the patients with DOC are nevertheless characterized by this lack of concordance between level of consciousness and behavioral responsivity. In our study, the proportion of responders found in the VS (10\%) and MCS(44\%) groups is in accordance with previous studies indicating that a minority of patients who do not show responses to command based on behavioral measures display signs of volitional mental activity when using active paradigms. ${ }^{2-4,6}$ It should be noted that the sensitivity of our ERP paradigm was not perfect because 3 out of 14 controls did not show a significantly higher P3 amplitude in the active condition. This is likely related to the simplicity of the task, which may have decreased amplitude differences between active and passive conditions.

P3 latencies appeared to be longer in MCS+ patients relative to controls. Whereas $\mathrm{P} 3$ amplitude reflects the level of attentional resource allocation (ie, attentional load), P3 


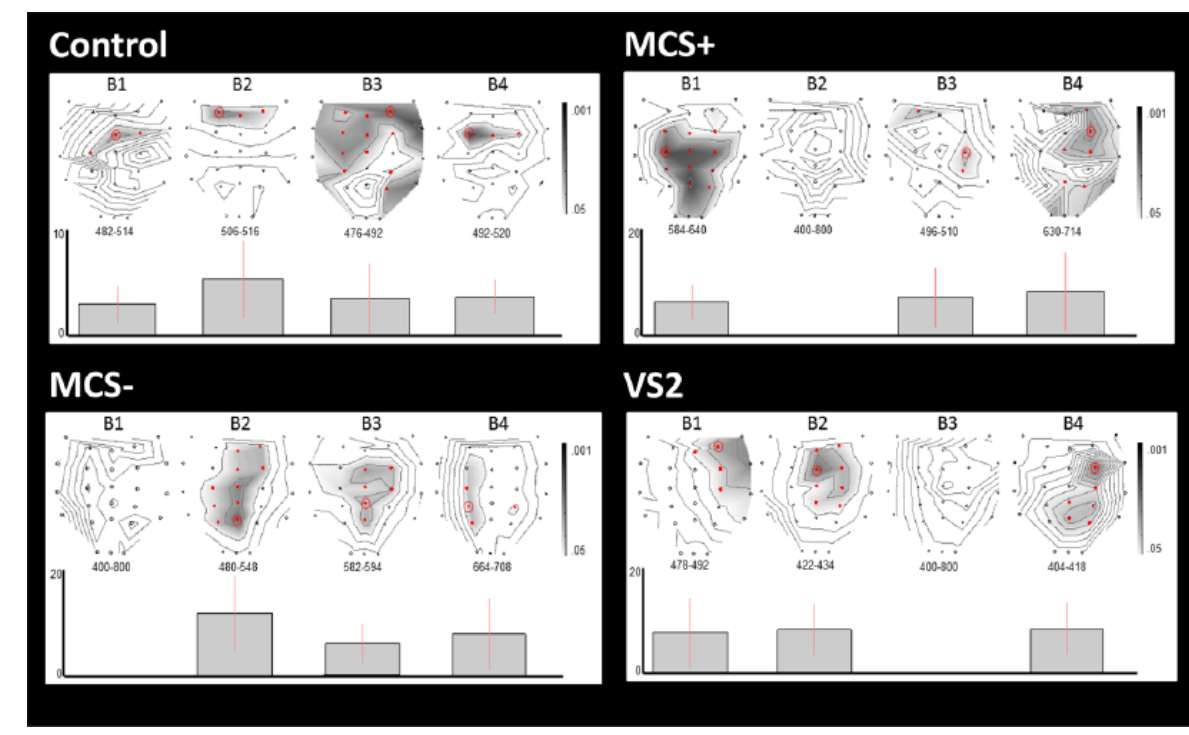

Figure 2. Differences in ERP responses across blocks. This figure illustrates the differences in P3 amplitude between active and passive conditions across blocks (BI-B4) for responders in the control group, the MCS+ group, the MCS- group, and for patient VS2. The upper part of each panel displays statistical parametric $T$ maps for the differences in amplitude between conditions $(P$ values $<.05$ to .001 in gray on the map; red circles are electrodes for which a significant result was observed) within the time window of interest (in ms; mentioned under the map). For illustrative purposes, the lower part of each panel represents the effect size of those differences (bars $=90 \%$ confidence intervals) for each block at the electrode where the largest effect (ie, lowest $P$ values) was observed (circled in red on the map).

Abbreviations: ERP, event-related potential; MCS, minimally conscious state; VS, vegetative state.

latency rather reflects the speed with which a target stimulus is detected and evaluated (ie, task difficulty). ${ }^{26} \mathrm{~A}$ longer P3 latency is in line with previous findings and suggests a slower cognitive processing compared with controls. ${ }^{5,44}$ Even though some MCS- patients showed a shorter P3 latency, responders in both MCS+ and MCS- groups showed similar longer latencies in the last block compared with the 2 first ones, suggesting a potential effect of fatigue. Such an observation cannot be made for patient VS2, who showed a consistent short latency across blocks, similar to that in controls. Because SPM software does not allow us to test for differences in latency, these assumptions are purely speculative. Future studies should investigate further the changes of latency over time as a potential indicator of fatigue. Besides, responders who were behaviorally classified as MCS- and VS were collapsed into 1 group to allow statistical analyses. Cognitive processing may differ among those groups. Future studies should investigate in a larger sample the remnant cognitive functioning in responsive patients diagnosed as being in a VS.

Our results have several limitations. Because the P3 latency varies widely between both individuals and groups, one could argue that the presumed P3 component could, in fact, reflect a different component. P3 latency is typically detected within a time window between 250 and $500 \mathrm{~ms}^{26}$ but may vary between 250 and $1000 \mathrm{~ms}$, depending on the stimulus and task applied. ${ }^{45}$ In fact, the P3 latencies found in the current study are parallel to those found in previous studies using the participant's own name paradigm in healthy volunteers ${ }^{20,46}$ as well as in severely brain-injured patients. ${ }^{5,44}$ Because the presence of the $\mathrm{N} 1$ component may not be obvious when looking at the grand-averaged ERPs or the single-subject averages, one could also argue that it is difficult to know whether our noncommunicative patients did hear the instruction or the stimulation. However, all patients presented at least an auditory startle (CRS-R auditory subscore $\geq 1$ ), which typically reflects the presence of auditory processing. ${ }^{47}$ Difficulty in seeing an N1 component may be a result of the high stimulus presentation frequency $(\mathrm{n}=100)$, which is known to dramatically reduce the N1-amplitude. ${ }^{48}$ Besides, in the patient groups, an N1 component could have been overshadowed by high latency heterogeneities that can be observed among severely braininjured patients ${ }^{44}$ but also by slow delta waves which are predominant in these patients. ${ }^{49,50}$ Controls were not matched to patients according to gender. P3 amplitude tends to be slightly larger in women than in men $(2$ to $3 \mu \mathrm{V}) .{ }^{26} \mathrm{In}$ our study, the only analysis directly comparing controls to patients included more female participants in the control group $(73 \%)$ than in the patients group $(40 \%$ in the MCS+ group and $50 \%$ in the CC group), which could potentially have led to a slight overestimation of the differences 


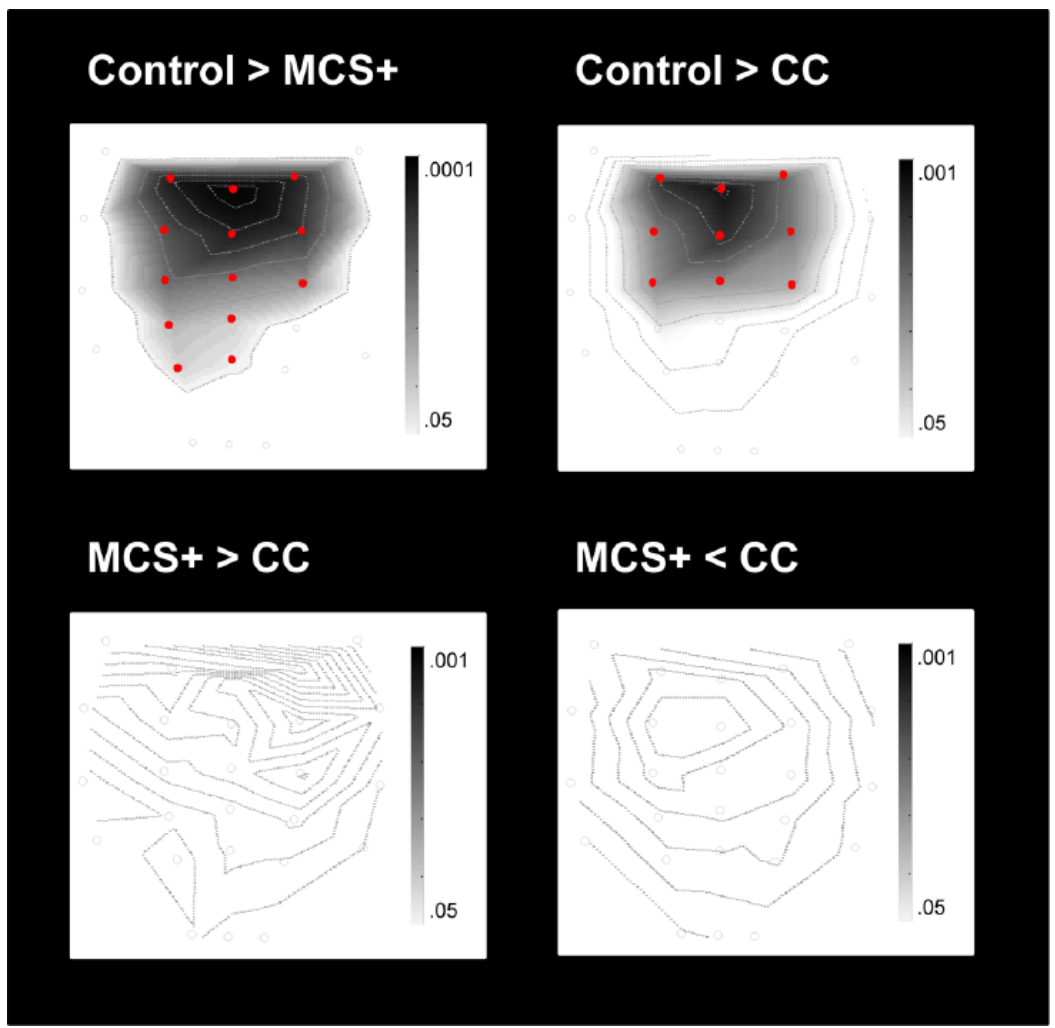

Figure 3. Differences in ERP responses between groups. This figure illustrates significantly higher $(>)$ or lower $(<)$ P3 amplitudes (in the active condition) when comparing groups (control, MCS+, and CC [covert cognition]). The statistical parametric T maps display the topography of those contrasts $(P$ values $<.05$ to $.00 \mathrm{I}$ in gray on the map; red circles are electrodes for which a significant result was observed) within the time window of interest ( $400-800$ ms poststimulus).

Abbreviations: ERP, event-related potential; MCS, minimally conscious state; VS, vegetative state.

between controls and patients. Finally, because covert cognition is present in a minority of severely brain-injured patients, our study only included a few patients. Future studies should include a very large sample and, besides replicating our results, should investigate the potential differences in cognition between patients diagnosed as being in a MCS- and patients diagnosed as being in a VS.

\section{Conclusion}

The findings of the current study suggest altered top-down attention in patients with electrophysiological signs of covert cognition. The results may have implications for the use of BCIs. The potential use of such technology as a means of restoring communication ability in severely braininjured patients has received increased attention. ${ }^{13-15}$ Monti et $\mathrm{al}^{2}$ observed yes-no communication using mental imagery in a severely brain-injured patient who was not able to communicate behaviorally. This phenomenon was observed in only 1 of 54 patients $(1.8 \%)$ studied. Lulé et al ${ }^{51}$ did not observe reliable yes-no communication in the 16 patients with DOC whom they assessed using a 4-choice auditory oddball EEG-BCI paradigm. In view of the potential impairments in top-down attentional processing observed in our study using a very simple task, it is likely that some patients with covert cognition will not be able to harness complex tasks such as mental imagery for communicative purposes. We believe that future BCIs should be simplified to improve the likelihood of eliciting an intelligible response. Whereas our findings require replication by independent investigators and in larger samples, future studies should also address other top-down cognitive processes (such as working memory, executive functioning, and episodic memory) and explore the interaction between these components and motor functions. Such studies could allow a better understanding of level and quality of residual top-down cognitive processing in severely brain-injured patients and help adapt BCI-based communication strategies to this population.

\section{Declaration of Conflicting Interests}

The authors declared no potential conflicts of interest with respect to the research, authorship, and/or publication of this article. 


\section{Funding}

The authors disclosed receipt of the following financial support for the research, authorship, and/or publication of this article: This work was supported by the National Funds for Scientific Research (A 4/5, MCF/DM) and the National Natural Science Foundation of China (30870861).

\section{Authors' Note}

CS and JTG contributed equally to the article. The data related to this study can be made available on request by the corresponding author.

\section{Supplemental Material}

Supplementary material for this article is available on the Neurorehabilitation \& Neural Repair Web site at http://nnr.sagepub.com/supplemental.

\section{References}

1. Owen AM, Coleman MR, Boly M, et al. Detecting awareness in the vegetative state. Science. 2006;313:1402.

2. Monti MM, Vanhaudenhuyse A, Coleman MR, et al. Willful modulation of brain activity in disorders of consciousness. $N$ Engl J Med. 2010;362:579-589.

3. Cruse D, Chennu S, Chatelle C, et al. Bedside detection of awareness in the vegetative state: a cohort study. Lancet. 2011;378:2088-2094.

4. Cruse D, Chennu S, Chatelle C, et al. Relationship between etiology and covert cognition in the minimally conscious state. Neurology. 2012;78:816-822.

5. Schnakers C, Perrin F, Schabus M, et al. Voluntary brain processing in disorders of consciousness. Neurology. 2008;71:1614-1620.

6. Faugeras F, Rohaut B, Weiss N, et al. Probing consciousness with event-related potentials in the vegetative state. Neurology. 2011;77:264-268.

7. Goldfine AM, Victor JD, Conte MM, et al. Determination of awareness in patients with severe brain injury using EEG power spectral analysis. Clin Neurophysiol. 2011;122:21572168.

8. Lechinger J, Chwala-Schlegel N, Fellinger R, et al. Mirroring of a simple motor behavior in disorders of consciousness. Clin Neurophysiol. 2013;124:27-34.

9. Monti MM, Cognition in the vegetative state. Annu Rev Clin Psychol. 2012;8:431-454.

10. The Multi-Society Task Force on PVS. Medical aspects of the persistent vegetative state. $N$ Engl J Med. 1994;330: 1499-1508.

11. Giacino JT, Ashwal S, Childs N, et al. The minimally conscious state: definition and diagnostic criteria. Neurology. 2002;58:349-353.

12. Nachev P, Hacker PM. Covert cognition in the persistent vegetative state. Prog Neurobiol. 2010;91:68-76.

13. Kübler A, Kotchoubey B. Brain-computer interfaces in the continuum of consciousness. Curr Opin Neurol. 2007;20:643-649.
14. Naci L, Monti MM, Cruse D, et al. Brain-computer interfaces for communication with nonresponsive patients. Ann Neurol. 2012;72:312-323.

15. Sorger B, Reithler J, Dahmen B, et al. A real-time fMRIbased spelling device immediately enabling robust motorindependent communication. Curr Biol. 2012;22:1333-1338.

16. Chennu S, Finoia P, Kamau E, et al. Dissociable endogenous and exogenous attention in disorders of consciousness. Neuroimage Clin. 2013;3:450-461.

17. Posner MI. Attentional networks and consciousness. Front Psychol. 2012;3:64.

18. Fan J, Gu X, Guise KG, et al. Testing the behavioral interaction and integration of attentional networks. Brain Cogn. 2009;70:209-220.

19. Laureys S, Schiff ND. Coma and consciousness: paradigms (re)framed by neuroimaging. Neuroimage. 2012;61:478-491.

20. Perrin F, García-Larrea L, Mauguière F, et al. A differential brain response to the subject's own name persists during sleep. Clin Neurophysiol. 1999;110:2153-2164.

21. Davis MH, Coleman MR, Absalom AR, et al. Dissociating speech perception and comprehension at reduced levels of awareness. Proc Natl Acad Sci U S A. 2007;104:1603216037.

22. Corbetta M, Shulman GL. Control of goal-directed and stimulus-driven attention in the brain. Nat Rev Neurosci. 2002;3:201-215.

23. Posner MI, Sheese BE, Odludas Y, et al. Analyzing and shaping human attentional networks. Neural Netw. 2006;19:1422-1429.

24. Wood N, Cowan N. The cocktail party phenomenon revisited: how frequent are attention shifts to one's name in an irrelevant auditory channel? J Exp Psychol Learn Mem Cogn. 1995;21:255-260.

25. Baluch F, Itti L. Mechanisms of top-down attention. Trends Neurosci. 2011;34:210-224.

26. Polich J. Updating P300: an integrative theory of P3a and P3b. Clin Neurophysiol. 2007;118:2128-2148.

27. Giacino JT, Kalmar K, Whyte J. The JFK Coma Recovery Scale-Revised: measurement characteristics and diagnostic utility. Arch Phys Med Rehabil. 2004;85:2020-2029.

28. Løvstad M, Frøslie KF, Giacino JT, et al. Reliability and diagnostic characteristics of the JFK coma recovery scale-revised: exploring the influence of rater's level of experience. $J$ Head Trauma Rehabil. 2010;25:349-356.

29. La Porta F, Caselli S, Ianes AB, et al. Can we scientifically and reliably measure the level of consciousness in vegetative and minimally conscious states? Rasch analysis of the Coma Recovery Scale-Revised. Arch Phys Med Rehabil. 2013;94:527-535.

30. Bruno MA, Majerus S, Boly M, et al. Functional neuroanatomy underlying the clinical subcategorization of minimally conscious state patients. J Neurol. 2012;259:1087-1098.

31. Klem GH, Luders HO, Jasper HH, et al. The ten-twenty electrode system of the International Federation. The International Federation of Clinical Neurophysiology. Electroencephalogr Clin Neurophysiol Suppl. 1999;52:3-6.

32. Herrmann CS, Knight RT. Mechanisms of human attention: event-related potentials and oscillations. Neurosci Biobehav. 2001;25:465-476. 
33. Polich J, Eischen SE, Collins GE. P300 from a single auditory stimulus. Electroencephalogr Clin Neurophysiol. 1994;92:253-261.

34. Mertens R, Polich J. P300 from a single-stimulus paradigm: passive versus active tasks and stimulus modality. Electroencephalogr Clin Neurophysiol. 1997;104:488-497.

35. Cass M, Polich J. P300 from a single-stimulus paradigm: auditory intensity and tone frequency effects. Biol Psychol. 1997;46:51-65.

36. Polich J, Margala C. P300 and probability: comparison of oddball and single-stimulus paradigms. Int J Psychophysiol. 1997;25:169-176.

37. Croft RJ, Gonsalvez CJ, Gabriel C, et al. Target-to-target interval versus probability effects on P300 in one- and twotone tasks. Psychophysiology. 2003;40:322-328.

38. Folmer RL, Yingling CD. Auditory P3 responses to name stimuli. Brain Lang. 1997;56:306-311.

39. Boly M, Garrido MI, Gosseries O, et al. Preserved feedforward but impaired top-down processes in the vegetative state. Science. 2011;332:858-862.

40. Katayama J, Polich J. Stimulus context determines P3a and P3b. Psychophysiology. 1998;35:23-33.

41. Schupp HT, Flaisch T, Stockburger J, et al. Emotion and attention: event-related brain potential studies. Prog Brain Res. 2006;156:31-51.

42. Petersen SE, Posner MI. The attention system of the human brain: 20 years after. Annu Rev Neurosci. 2012;35:73-89.

43. Bruno MA, Vanhaudenhuyse A, Thibaut A, et al. From unresponsive wakefulness to minimally conscious PLUS and functional locked-in syndromes: recent advances in our understanding of disorders of consciousness. J Neurol. 2011;258:1373-1384.
44. Perrin F, Schnakers C, Schabus M, et al. Brain response to one's own name in vegetative state, minimally conscious state, and locked-in syndrome. Arch Neurol. 2006;63: 562-569.

45. Duncan CC, Barry RJ, Connolly JF, et al. Event-related potentials in clinical research: guidelines for eliciting, recording, and quantifying mismatch negativity, P300, and N400. Clin Neurophysiol. 2009;120:1883-1908.

46. Eichenlaub JB, Ruby P, Morlet D. What is the specificity of the response to the own first-name when presented as a novel in a passive oddball paradigm? An ERP study. Brain Res. 2012;1447:65-78.

47. Yeomans JS, Frankland PW. The acoustic startle reflex: neurons and connections, Brain Res Rev. 1995;21:301-314.

48. Knight RT, Hillyard SA, Woods DL, et al. The effects of frontal and temporal-parietal lesions on the auditory evoked potential in man. Electroencephalogr Clin Neurophysiol. 1980;50:112-124.

49. Leon-Carrion L, Martin-Rodriguez JF, Damas-Lopez J, et al. Brain function in the minimally conscious state: a quantitative neurophysiological study. Clin Neurophysiol. 2008;119: 1506-1514.

50. Lehembre R, Bruno MA, Vanhaudenhuyse A, et al. Restingstate EEG study of comatose patients: a connectivity and frequency analysis to find differences between vegetative and minimally conscious states. Funct Neurol. 2012;27: 41-47.

51. Lulé D, Noirhomme Q, Kleih SC, et al. Probing command following in patients with disorders of consciousness using a brain-computer interface. Clin Neurophysiol. 2013;124: 101-106. 